\title{
A finite number of regular rotational bands in the superdeformed well of ${ }^{143} \mathrm{Eu}$
}

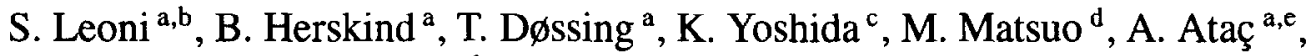 \\ G.B. Hagemann ${ }^{\text {a }}$, F. Ingebretsen ${ }^{f}$, H.J. Jensen ${ }^{\text {a }}$, R.M. Lieder ${ }^{\text {a,g }}$, G.V. Marti ${ }^{g}$, N. Nica ${ }^{\text {a, }}$, \\ J. Nyberg ${ }^{a, i}$, M. Piiparinen ${ }^{a, j}$, H. Schnare ${ }^{g}$, G. Sletten ${ }^{a}$, K. Strähle ${ }^{g}$, M. Sugawara ${ }^{\text {a,k }}$, \\ P.O. Tjøm ${ }^{\mathrm{f}}$, A. Virtanen ${ }^{\mathrm{a}, \mathrm{j}}$ \\ ${ }^{a}$ The Niels Bohr Institute, University of Copenhagen, Copenhagen, Denmark \\ ${ }^{b}$ Department of Physics, University of Milano and INFN Milano, Milano, Italy \\ c Department of Physics, Kyoto University, Kyoto, Japan \\ d Yukawa Institute for Theoretical Physics, Kyoto University, Kyoto, Japan \\ e Department of Radiation Sciences, Uppsala University, Uppsala, Sweden \\ ${ }^{f}$ Department of Physics, University of Oslo. Oslo, Norway \\ g Institut für Kernphysik, K.F.A. Jülich, Jülich, Germany \\ ${ }^{\mathrm{h}}$ Heavy Ion Department, Institute of Atomic Physics, Bucharest, Rumania \\ i The Svedberg Laboratory, Uppsala University, Uppsala, Sweden \\ j Department of Physics, University of Jyväskylä, Jyväskylä, Finland \\ k Chiba Institute of Technology, Shibazono, Narashino 275, Japan
}

Received 21 February 1995; revised manuscript received 7 April 1995

Editor: R.H. Siemssen

\begin{abstract}
The number of excited superdeformed bands in ${ }^{143} \mathrm{Eu}$ is measured by use of the Fluctuation Analysis Method. Between 10 and 40 rotational bands, displaying typical rotational energy correlations over two consecutive transitions, are populated within a rather narrow range in transition energy, $E_{\gamma} \approx 1300-1500 \mathrm{keV}$. These numbers are close to the values found for normally deformed nuclei and agree with microscopic cranking + band mixing calculations for the specific superdeformed nucleus, which predict the onset of rotational damping to occur at the excitation energy $U_{0}=1.3-1.6 \mathrm{MeV}$ above the yrast line.
\end{abstract}

Although discrete spectroscopy is very powerful for studying nuclear rotational motion at low excitation energy, it fails in the region of higher level density where the rotational transition strength becomes fragmented due to the residual interaction, corresponding to a damping of the rotational motion [1]. This implies very complex sequences of levels, connected by weak $\gamma$-lines which cannot be resolvcd, even with the improved spatial resolution obtained from higher fold $\gamma$-coincidences (measured $\gamma$ multiplicity). Therefore a statistical method was developed recently to extract more general information about the level structure in the quasi-continuum region. From the analysis of the fluctuation of counts in specific sections of the $E_{\gamma_{1}} \times E_{\gamma_{2}}$ coincidence spectra using the Fluctuation Analysis Method (FAM) [2-4], quantitative estimates of the number of rotational bands participating in the decay process can be obtained, both for the 
damped region and for the region where regular rotational motion is dominating. From our knowledge about level densities the information can in turn also be used to estimate the excitation energy where the onset of the damped motion occurs. The method was extensively used to study the rotational decay of several normally deformed nuclei in the rare earth region [3,5]. It was confirmed that the well known pattern of ridge structures flanking the central valley at $E_{\gamma_{1}}=$ $E_{\gamma_{2}}$ in $E_{\gamma_{1}} \times E_{\gamma_{2}}$ spectra mainly consists of low lying regular rotational bands. The effective number of bands is generally found for the rare earth region to be of the order of $\approx 40$, which roughly covers a range in excitation energy from yrast up to $U_{0} \approx 800 \mathrm{keV}$, the onset energy for rotational damping. In fact, above this region rotational damping becomes progressively dominant and the rotational decay is fragmented over a huge number of paths determined by FAM to be $\approx 10^{4}$. For the nucleus ${ }^{168} \mathrm{Yb}[6]$, these experimental estimates are in agreement with cranked shell model and band mixing calculations with the inclusion of residual interactions.

While a rather consistent picture of the rotational decay in normally deformed nuclei starts to emerge, very little is known about the quasi-continuum in highly deformed nuclei. A large number of superdeformed yrast bands has been found in recent years, and information about several excited bands in specific nuclei has been obtained. In a few nuclei a superdeformed (SD) ridge structure has been observed also and has been found to appear in a rather narrow spin range, with an intensity only 3 times bigger than the full intensity of the SD yrast transitions $[7,8]$.

In the present work we apply for the first time the FAM technique to the analysis of the quasi-continuum in a SD nucleus, ${ }^{143} \mathrm{Eu}$. This provides an experimental estimate of the number of discrete rotational bands in the SD well. The results will be compared to realistic band mixing calculations for the specific nucleus.

A high statistics and high fold data set on ${ }^{143} \mathrm{Eu}$ was obtained at the Niels Bohr Institute Tandem Accelerator Laboratory using the NORDBALL detector array equipped with 19 Compton-suppressed Ge detectors, $1 \mathrm{Ge}$ LEP (Low Energy Photon) detector and a $60 \mathrm{el}$ ements $\mathrm{BaF}_{2}$ scintillator inner-ball for multiplicity and sum-energy selection. The states in ${ }^{143} \mathrm{Eu}$ were populated by the reaction ${ }^{110} \mathrm{Pd}\left({ }^{37} \mathrm{Cl}, 4 n\right){ }^{143} \mathrm{Eu}$ at a beam energy of $160 \mathrm{MeV}$. The target consisted of 2-3 self-

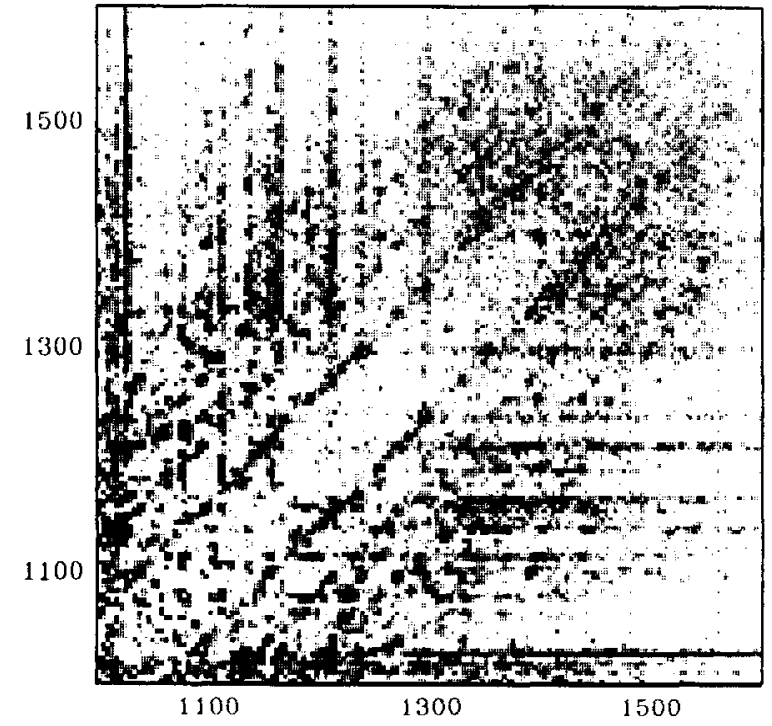

Fig. 1. The figure shows the $E_{\gamma_{1}} \times E_{\gamma_{2}}$ coincidence matrix of ${ }^{143} \mathrm{Eu}$ in the transition energy region $1000-1600 \mathrm{keV}$. The spectrum has been background subtracted using the COR treatment [10,5]. One can clearly observe the presence of a ridge structure running parallel to the $E_{\gamma_{1}}=E_{\gamma_{2}}$ diagonal, with a spacing of $60 \mathrm{keV}$ from the diagonal and between the first and second ridge, corresponding to the average dynamic moment of inertia $3^{(2)}$ of the yrast SD band.

supporting foils isotopically enriched to $98.6 \%$ and the total target thickness was $1.2 \mathrm{mg} / \mathrm{cm}^{2}$. A total of $10^{9}$ triple and higher fold coincidence events was collected. The spectra were cleaned from delayed $\gamma$-rays and a significant fraction of neutron induced peaks by setting a narrow energy dependent time gate on the Ge energies. After requiring a high fold $(\geq 12)$ and high sum-energy in the $\mathrm{BaF}_{2}$ detectors, the remaining $500 \times 10^{6}$ three and higher fold events were stored into a $0.5 \times 0.5(\mathrm{keV} / \text { channel })^{2}$ coincidence matrix [9].

Although the population of the SD yrast band is of the order of only $1 \%$ of the intensity populating ${ }^{143} \mathrm{Eu}$, a ridge structure with a spacing of $4 / \Im^{(2)}=60 \mathrm{keV}$, corresponding to the second moment of inertia of the SD band, is observed very clearly, as shown in the $\gamma-\gamma$ matrix of Fig. 1. The matrix was background subtracted with the COR treatment [10,5]. Fig. 2(a) shows the relative intensities of the SD yrast transitions (squares) and of the first and second ridge (closed and open circles) analyzed in the region free from disturbing lines from the normal deformed states. One can observe that the feeding into the excited bands 


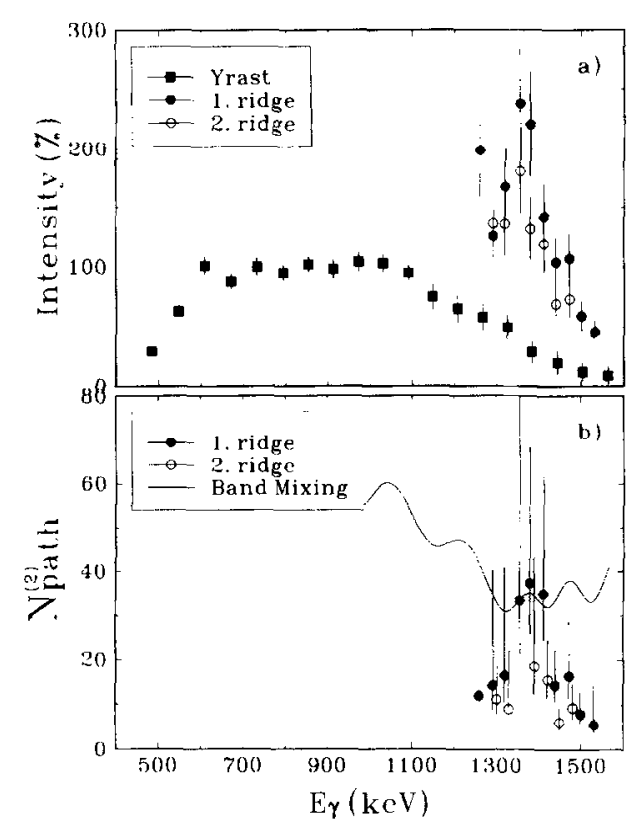

Fig. 2. The upper part of the figure shows the relative intensities of the yrast SD transitions (squares), normalized to an average of the highest points. The closed circles and the open circles represent the intensities of the first and second ridges, respectively. The values of $N_{\text {path }}^{(2)}$ deduced for the first and second superdeformed ridge structures in ${ }^{143} \mathrm{Eu}$ for intervals of $60 \mathrm{keV}$ width along the ridges, in steps of $30 \mathrm{keV}$, are shown in the bottom part. The fluctuations in the second ridge at $1350 \mathrm{keV}$ could not be extracted due to a close lying peak from the first well. The values were deduced after all coincidence peaks from the known superdeformed yrast band have been removed from the COR spectrum shown in Fig. 1. The curve represents the prediction for the first ridge from cranked shell model calculations with inclusion of SDI residual interaction [6].

takes place in a rather narrow transition energy region around $1400 \mathrm{keV}$, slightly higher than that into the SD yrast band, and that the second ridge appears to be roughly $40 \%$ weaker than the first one. Since the first ridge is almost a factor of 10 larger in intensity than the yrast transitions at the same transition energy we can already place a lower limit on the number of bands. At least 10 excited bands of intensity comparable to the SD yrast band must exist. One can observe that the general features of the ridge structure in ${ }^{143} \mathrm{Eu}$ are rather similar to the ones observed earlier in ${ }^{152} \mathrm{Dy}$ [7].

The fluctuation analysis method was applied to the data to obtain a more precise estimate of the number of excited regular SD bands populating the ridges.
The method is based on the well known property that the larger the number of choices (in $E_{\gamma_{1}} \times E_{\gamma_{2}}$ ) available for each event, the smaller the associated fluctuations in the number of counts will be. This implies that strong fluctuations will be associated with the few cold discrete rotational bands near yrast, typically producing rather spiky ridges in the $E_{\gamma_{1}} \times E_{\gamma_{2}}$ spectrum. In contrast, weaker fluctuations will be produced by the transitions from the warmer quasi-continuum region, which spread out more in the plane $E_{\gamma_{1}} \times E_{\gamma_{2}}$ and produce a smooth spectrum. A very simple expression relates the effective number of paths to the count fluctuations in the spectrum $[2,3]$ :

$N_{\text {path }}^{(2)}=\frac{N_{\text {eve }}}{\frac{\mu_{2}}{\mu_{1}}-1} \frac{P^{(2)}}{P^{(1)}}$

Here $\mu_{1}$ and $\mu_{2}$ are the first and second moments of the number of counts in the sector $4 / \Im^{(2)} \times 4 / \Im^{(2)}$, in which each cascade contributes on the average one count. $N_{\text {eve }}$ is the number of events in the sector and $P^{(2)} / P^{(1)}$ is a correction factor due to the finite resolution of the experiment.

The moments $\mu_{1}$ and $\mu_{2}$ of the spectrum were extracted from the 2-D spectrum by the program STATFIT [3,5], after the known discrete lines from the SD yrast band were removed from the $E_{\gamma_{1}} \geq E_{\gamma_{2}}$ part of the high resolution matrix $(0.5 \mathrm{keV} / \mathrm{channel})$ by means of a 2-D interactive Gaussian fitting program, called " 42 " [11]. It has been shown that the removal of known lines which are especially intense relative to the yet unresolved transitions in the ridges makes the estimate of the number of pathways more accurate [3]. By removing only the lines in one of the symmetrical sectors of the 2-D spectrum, it is possible to check, and in the present case confirm, the statistical independence between the energies of the yrast SD band and those of the bands contributing to the ridge.

The left and right panels of Fig. 3 show typical perpendicular cuts on the $\mu_{1}$ and $\mu_{2}$ spectra, with 4 $\mathrm{keV} /$ channel dispersion and for 5 different transition energies intervals over the range $1230-1530 \mathrm{keV}$. It looks like the ridge structures extend further down in transition energy (see Fig. 1), but impurities from intense transitions in the first well prohibited a meaningful analysis. The width of the cuts is given by the measured distance between the SD ridges, $60 \mathrm{keV}$, and corresponds to an average dynamic moment of inertia 


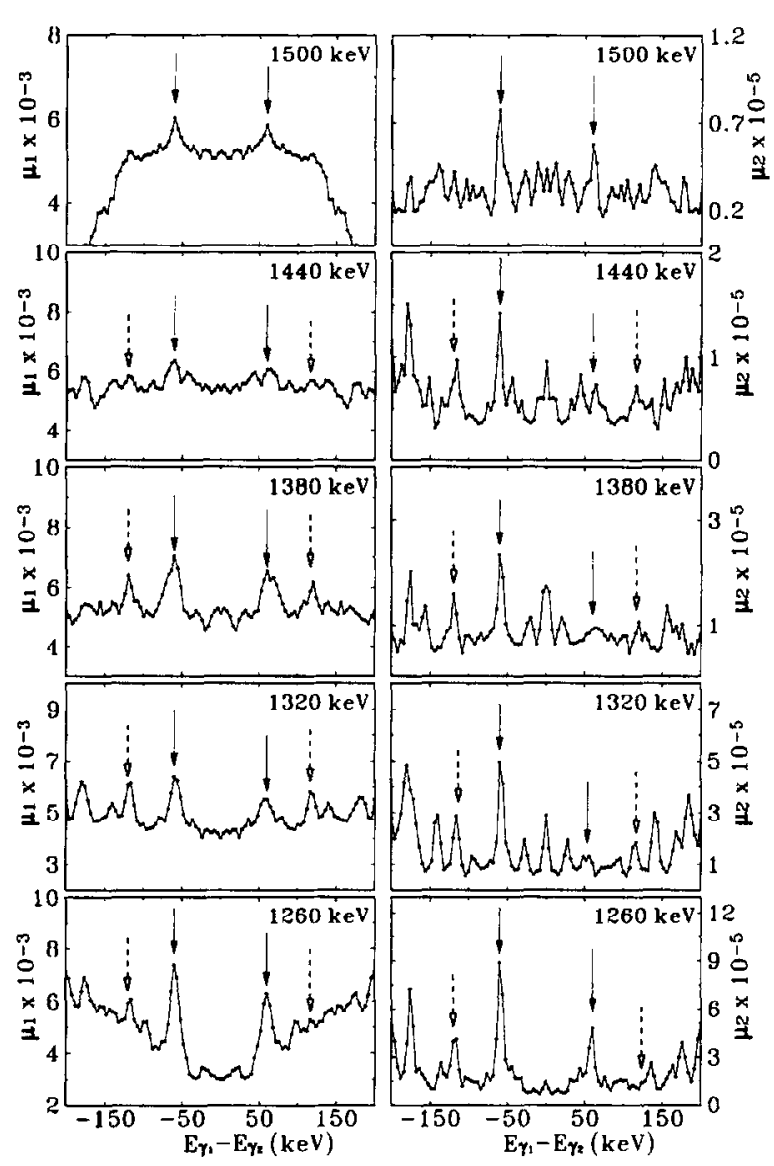

Fig. 3. Perpendicular cuts, $60 \mathrm{keV}$ wide, of the moments $\mu_{1}$ and $\mu_{2}$ of the COR spectrum shown in Fig. 1, for 5 different average transition energies in the region $1230-1530 \mathrm{keV}$. The solid (dashed) arrows point at the place of the first (second) ridge positions. The yrast line subtraction is clearly visible in the reduced ridge intensity in the $E_{\gamma_{1}} \geq E_{\gamma_{2}}$ part of the intensity spectra.

of $66.7\left(\hbar^{2} / \mathrm{MeV}\right)$. The location of the first and second ridge are indicated by arrows. One can observe that the second ridge is weaker than the first, as also seen in Fig. 1. The results of the subtraction of yrast peaks can be clearly seen from the reduced intensities in the first and second ridges in the $E_{\gamma_{1}} \geq E_{\gamma_{2}}$ part of the $\mu_{1}$ spectrum. For all the energy intervals, the first ridge is clearly recognized in the $\mu_{1}$ intensity spectra. In the $\mu_{2}$ spectra, the fluctuations from the SD yrast band strongly dominate for $E_{\gamma_{1}} \leq E_{\gamma_{2}}$, while the remaining first ridge seen in the $E_{\gamma_{1}} \geq E_{\gamma_{2}}$ part of the spectra carries much smaller fluctuations especially for energies $E_{\gamma} \geq 1300 \mathrm{keV}$. According to Eq. (1), this gives an increase in the number of bands around $\approx 1350 \mathrm{keV}$.

The average value of $N_{\text {path }}^{(2)}$ shown in Fig. $2(\mathrm{~b})$ is $\approx$ 20 and about 2 times smaller than observed for normally deformed nuclei [2]. It is interesting to note that around $1350 \mathrm{keV}$ a maximum of effective number of bands of 40 carries about $2 \%$ of the total decay flow in ${ }^{143} \mathrm{Eu}$, which means that each excited band is on average populated by an intensity of $0.05 \%$. The relatively large error bars are significantly bigger than the "principle error" given by the counter resolution (typically $20 \%$ ) $[3,4]$. This is caused by the extremely weak absolute intensity of the SD ridge compared to the statistical fluctuations in the background. It is likely that the most of these bands belong to ${ }^{143} \mathrm{Eu}$, since the excitation energy was optimized for populating the SD yrast of ${ }^{143} \mathrm{Eu}$, and more than $70 \%$ of the total population goes into ${ }^{143} \mathrm{Eu}$ for fold grater than 12 .

The experimental estimate of the number of paths can be compared to results from a theoretical cranked shell model calculation. In the latter, diagonalization for multi-particle multi-hole states was performed and a residual Surface Delta Interaction (SDI) was included $[16,6]$. This was done for the specific nucleus ${ }^{143} \mathrm{Eu}$. The number of undamped rotational bands populating the first ridge has been obtained by counting all the bands stretching over more than two consecutive transitions, with a branching number $n_{\text {branch }}=$ $1 / \Sigma_{j} w_{i j}^{2}<2$, where $w_{i j}$ denotes the normalized rotational transition strength from the level $i$ at spin $I$ to the level $j$ at spin $I-2$. The branching number gives a good estimate of the branching of the decay from each state and it is often used to define the onset of damping, by the condition $n_{\text {branch }}>2$ [3]. The results obtained for the first ridge are shown by the curve of Fig. 2(b). As shown, the calculation reproduces the data at the maximum of the distribution, while it deviates strongly at higher and lower transition energies. This is generally expected for the lower part of the ridge around $1300 \mathrm{keV}$ [12], since barrier penetration to the first well removes intensity from the excited bands, eventually leaving only the yrast band together with a few low lying bands to survive further down in angular momentum. The decrease observed in the number of bands at the highest transition energies falls in the region where fission competition is important at higher excitation energies. However, it is not straightforward to understand the reduction in the number of populated bands in the fission dominated 
region without a further study of the population mechanism in this regime. The lower number of decay paths observed for the second ridge is in good accordance with the damping picture. The progressive fragmentation of the rotational strength implies that a smaller number of decay paths can keep rotational energy correlations over three steps relative to two steps [13].

The microscopic calculations predict that the onset of damping for ${ }^{143} \mathrm{Eu}$ occurs at $1.3-1.6 \mathrm{MeV}$ above yrast, which is much higher than observed in normally deformed nuclei. This is in agreement with earlier investigations based on simulations of the intensities observed in the ridge structures of ${ }^{152}$ Dy [14], and caused by the considerably smaller level density expected in the SD well [15].

We can finally conclude that the fluctuation analysis method predicts the existence of up to 40 excited superdeformed bands in ${ }^{143} \mathrm{Eu}$, before rotational damping starts to be important in the second well. This implies that each of the excited bands is on average populated by an intensity of $\approx 0.05 \%$, which will be within reach by use of the new generation of $4 \pi$-Ge detectors arrays. The number of discrete superdeformed rotational bands is close to the one found in normal deformed nuclei of the rare earth region and agrees with microscopic band mixing calculations for the specific nucleus. Altogether this indicates that damping sets in at $1.3-1.6 \mathrm{MeV}$ above yrast.
This work has been supported by the Danish Natural Science Research Council, the Swedish Natural Science Research Council and the Academy of Finland.

\section{References}

[1] B. Lauritzen, T. Døssing and R.A. Broglia, Nucl. Phys., A 457 (1986) 61-83.

[2] B. Herskind et al., Phys. Rev. Lett. 68 (1992).

[3] T. Døssing et al., accepted by Physics Reports.

[4] B. Herskind et al., Proc. Intern. Sem. on The Frontier of Nuclear Spectroscopy (Kyoto) (World Scientific, Singapore, 1993) p. 17.

[5] S. Leoni, Ph.D. Thesis, University of Milano (1992).

[6] M. Matsuo et al., Phys. Rev. Lett. 70 (1993) 2694.

[7] P.J. Twin, Proc. Intem. Conf. on Nuclear Shapes (Crete) (World Scientific, Singapore, 1988) p. 152.

[8] P.J. Twin, Nucl. Phys. A 574 (1994) 51c-72c, and reference therein.

[9] A. Ataç et al., Phys. Rev, Lett. 70 (1993) 1069.

[10] O. Andersen et al., Phys. Rev. Lett. 43 (1979) 687

[11] M. Bergström and P. Ekström, Nucl. Inst. and Meth. A 301 (1991) 132

[12] B. Herskind et al., Phys. Rev. Lett. 59 (1987) 2416.

[13] B. Herskind et al., Proc. Int. School on Heavy Ions Phys. III course (Erice), (World Scientific, Singapore, 1994) p. 197.

[14] K. Schiffer and B. Herskind, Phys. Lett. B 255 (1991) 508.

[15] S. Åberg, Phys. Rev. Lett. 64 (1990) 319.

[16] K. Yoshida and M. Matsuo, in preparation. 